\title{
Anti-Neutrophil Cytoplasmic Antibody-Associated Vasculitis in Mexican Population: Clinical Phenotype, Autoantibody Profile and Renal Characteristics
}

Andrea Hinojosa-Azaola, Carlos A. Nuñez-Alvarez, Christian Mauriel Uriarte-Hernández, José Luis García-Hernández and Jorge Alcocer-Varela* Department of Immunology and Rheumatology, Instituto Nacional de Ciencias Médicas y Nutrición Salvador Zubirán, Mexico City, Mexico

*Corresponding author: Jorge Alcocer-Varela, Department of Immunology and Rheumatology, Instituto Nacional de Ciencias Médicas y Nutrición Salvador Zubirán, Mexico City, Mexico, Tel: 52555655-5954; E-mail: jorgealcocer2@gmail.com

Received date: May 06, 2016; Accepted date: June 06, 2016; Published date: June 09, 2016

Copyright: ( 2016 Hinojosa-Azaola A, et al. This is an open-access article distributed under the terms of the Creative Commons Attribution License, which permits unrestricted use, distribution, and reproduction in any medium, provided the original author and source are credited.

\begin{abstract}
Objective: To describe the clinical, serological characteristics, and renal histology in Mexican patients with antineutrophil cytoplasmic antibody (ANCA) associated vasculitis (AAV).

Methods: Forty patients with diagnosis of AAV and renal biopsy, followed at a tertiary care center in Mexico City from 2002-2012 were retrospectively studied. Demographics, comorbidities, clinical, laboratory variables, disease activity, mortality, renal variables and histological classes were analyzed. Additionally, 50 patients with AAV, and a control group (other systemic vasculitis, systemic lupus erythematosus, infections, healthy subjects) were studied transversally to assess the prevalence of specific antibodies (ANCA, MPO, PR3, cathepsin G, lysozyme, lactoferrin, $\mathrm{BPI}$ and elastase), and their association with clinical manifestations.
\end{abstract}

Results: Granulomatosis with polyangiitis (GPA) was the predominant phenotype, and median age at diagnosis was 53 years. High disease activity (BVAS/GPA=24), general symptoms $(98 \%)$, renal $(80 \%)$, pulmonary $(63 \%)$, and ear, nose and throat (ENT) $(58 \%)$ involvement were frequent at diagnosis. Renal damage was present at diagnosis (serum creatinine $3.9 \mathrm{mg} / \mathrm{dl}$, estimated glomerular filtration rate $16 \mathrm{ml} / \mathrm{min}, 24 \mathrm{hr}$ proteinuria $2.5 \mathrm{~g}, 15 \%$ on dialysis). The most prevalent renal histological class was sclerotic $(58 \%)$, followed by mixed $(32 \%)$, focal ( $8 \%)$, and crescentic $(2 \%)$. No significant differences were found between histological classes in comorbidities, clinical, renal and laboratory variables, or survival. The main ANCA immunofluorescence pattern was C-ANCA (68\%), followed by XANCA (22\%), and P-ANCA (18\%). PR3-ANCA (high sensitive) was the most frequent antibody, positive in $86 \%$, while positivity for MPO-ANCA was observed in 18\%. ANCA positivity was more frequent in patients with AAV than the control group $(p<0.001)$. Antibodies directed to minor targets were infrequent in patients with AAV ( $2 \%)$. No significant correlations were identified between autoantibodies and clinical manifestations.

Conclusion: GPA phenotype, C-ANCA and PR3-ANCA positivity, high disease activity, chronic renal damage, general symptoms, ENT and lung involvement at diagnosis distinguish our population with AAV.

Keywords: ANCA; Vasculitis; Mexican; Ethnicity; Clinical phenotype; Autoantibodies; Renal histology

\section{Introduction}

Anti-neutrophil cytoplasmic antibody (ANCA) associated vasculitides (AAV) are infrequent, chronic, multi systemic autoimmune diseases characterized by the presence of vasculitis and necrosis affecting predominantly small vessels. The main phenotypes of AAV are granulomatosis with polyangiitis (GPA), microscopic polyangiitis (MPA), and eosinophilic granulomatosis with polyangiitis (EGPA). Disease patterns including clinical manifestations, serology and disease activity may vary among different regional and ethnic backgrounds.

Geographical differences in the incidence and expression of AAV have been demonstrated in Japan, Europe and North America [1]. The incidence and the prevalence of GPA are significantly higher in northern Europe (Norway and Germany), while MPA is predominant is southern Europe (Spain) [2]. Annual incidence of AAV is similar between Japan and UK (around 22/million adults). In Japan, MPA is the main subtype (83\%), whereas GPA is more frequent in UK (66\%), following the latitude theory of AAV. Moreover, P-ANCA and/or MPO-ANCA positivity predominates in Japanese patients $(>80 \%)$, whereas two-thirds of patients in the UK are C-ANCA and/or PR3ANCA positive [1]. African Americans with pauci-immune glomerulonephritis are younger and more often MPO-ANCA positive compared to Caucasians [3]. A study from China demonstrated that patients with GPA were predominantly MPO-ANCA positive and this antibody was associated with multiorgan involvement and higher levels of serum creatinine than PR3-ANCA patients with GPA [4].

Antibodies specific for other neutrophil antigens, such as cathepsin G, lysozyme, lactoferrin, bacterial permeability increasing protein (BPI), and elastase have been observed in specific clinical scenarios. Anti-elastase are present in drug-associated vasculitis and in cocaineinduced nasal destruction. Anti-BPI is present in C-ANCA-positive patients that are negative for MPO-ANCA and PR3-ANCA antibodies. Anti-cathepsin G has been found in Sjögren's syndrome and pediatric GPA. Anti-lactoferrin and anti-lysozyme can be present in druginduced vasculitis and other autoimmune diseases [5,6]. The low prevalence of these antibodies that are not routinely determined has limited conclusions regarding their clinical significance. 
Citation: Hinojosa-Azaola A, Nuñez-Alvarez CA, Uriarte-Hernández CM, García-Hernández JL, Alcocer-Varela J, et al. (2016) Anti-Neutrophil Cytoplasmic Antibody-Associated Vasculitis in Mexican Population: Clinical Phenotype, Autoantibody Profile and Renal Characteristics. J Vasc 2: 110. doi:10.4172/2471-9544.100110

Page 2 of 7

Differences in clinical phenotypes have also been described. For example, there is lower frequency of renal involvement in Japanese patients with GPA (12-63\%) compared to over 70\% in patients with GPA from Germany and USA [1]. The severity of kidney damage, as well as differences in therapy, influence the distribution of the renal histopathological classes among populations, as shown in the studies that have validated the histopathological classification of ANCAassociated glomerulonephritis proposed by Berden et al. [7,8]. Also, variations in the prevalence of interstitial lung disease between Asia and Europe have been described, with a higher prevalence of this manifestation in Asian countries probably associated with the predominance for MPO-ANCA positivity in this geographic region [2].

Few studies have assessed the characteristics of Mexican patients with AAV [9-12]. A study that compared Hispanics (Mexican ancestry) and Caucasians with AAV living in the same geographical area in Chicago showed that Hispanics present with a more severe disease activity, more damage, and a higher prevalence of renal involvement and kidney insufficiency requiring dialysis than Caucasians [13].

Because AAV is infrequent in non-Caucasians, and differences in disease expression among ethnic backgrounds exist, we aimed to describe the clinical and serological characteristics, as well as renal histology in Mexican patients with AAV from a single center.

\section{Patients and Methods}

\section{Clinical and renal histologic characteristics}

We retrospectively studied all patients with diagnosis of AAV, followed at the Department of Immunology and Rheumatology of the Instituto Nacional de Ciencias Médicas y Nutrición Salvador Zubirán, a tertiary care center in Mexico City, from January 2002 to December 2012. Our center is a referral Institution for mainly uninsured patients from all over the country. Inclusion criteria were: diagnosis of GPA or MPA according to definitions from the 2012 revised Chapel Hill Consensus Conference [14], availability of a kidney biopsy, and positivity for ANCA, MPO or PR3-ANCA antibodies. Information was retrieved from the medical records. Patients with EGPA, secondary forms of vasculitis, anti-glomerular basement membrane disease, lupus nephritis or IgA-nephropathy, and patients with missing data were excluded. Patients that had diagnosis of GPA or MPA and diabetes mellitus with a renal biopsy showing diabetic nephropathy were also excluded.

Demographics and comorbidities were considered at diagnosis of vasculitis. Clinical and laboratory variables were also analyzed at the time of AAV diagnosis, and included: antibodies (ANCA by indirect immunofluorescence assay, anti-PR3 and anti-MPO by ELISA), erythrocyte sedimentation rate (ESR), and high sensitivity C-reactive protein (hsCRP). Organ manifestations were only registered if symptoms could be ascribed to active vasculitis. Disease activity was scored using the Birmingham Vasculitis Activity Score specific for GPA (BVAS/GPA) [15], while prognosis was assessed using the Five-Factor Score (FFS) at diagnosis [16]. Mortality and causes of death were recorded during follow-up.

Renal variables included, serum creatinine, 24-hour urine protein excretion, protein/creatinine ratio, estimated glomerular filtration rate (eGFR), using MDRD formula [17], and dialysis requirements at diagnosis of AAV.
Forty patients with renal biopsy were included. In every case, one section of the kidney biopsy core was formalin fixed and paraffin embedded, $2 \mu \mathrm{m}$ sections were stained with haematoxylin and eosin (H\&E), periodic acid-Schiff, Masson's trichrome stain and Jone's methenamine silver stain. Direct immunofluorescence was performed on $3 \mu \mathrm{m}$ frozen sections that were exposed to antibodies against IgG, IgM, IgA, kappa, lambda, C1q, C3c, albumin and fibrinogen. All biopsies were reviewed and scored by two pathologists who were unaware of the clinical and laboratory information. Based on the pathological classification of ANCA-associated glomerulonephritis [7], all biopsies were evaluated and classified according to one of the four main categories (i.e. focal, crescentic, mixed or sclerotic). The percentage of normal glomeruli, tubular atrophy, interstitial infiltrate and interstitial fibrosis were also assessed.

\section{Autoantibodies}

From June 2014 to June 2015, 50 consecutive outpatients with diagnosis of AAV (GPA, MPA, EGPA or renal-limited vasculitis) according to definitions from the 2012 revised Chapel Hill Consensus Conference [14] were recruited at the Rheumatology consult of our Institution. The comparative control group comprised 10 patients with systemic vasculitis other than AAV (7 with takayasu's arteritis, 2 with behçet's disease and 1 with crioglobulinemia); 10 patients with systemic lupus erythematosus (SLE); 10 patients with infections without autoimmune disease (2 with tuberculosis, 3 with HIV/AIDS, 1 with bacterial endocarditis and 4 with bacterial infections), and 13 healthy subjects.

Evaluation of the patients with AAV included disease activity (BVAS/GPA), physician's global assessment (PGA), laboratory tests and treatment information.

All serum samples were tested for ANCA-IgG by indirect immunofluorescence (IIF) on ethanol fixed human neutrophils (NOVA Lite ANCA; INOVA Diagnostics, San Diego, USA). Serum screening dilution was 1:20 (Beeline 220s, HTZ; London, UK). Staining patterns were identified as perinuclear (P-ANCA), granular cytoplasmic (C-ANCA) and delineated atypical (X-ANCA). Atypical or P-ANCA patterns were confirmed in formalin fixed neutrophils substrate and antinuclear antibodies were ruled-out (INOVA Diagnostics, San Diego, USA).

Specific autoantibodies (IgG isotype) included: MPO, PR3 (QUANTA Lite; INOVA Diagnostics, San Diego, USA), high sensitivePR3, cathepsin G, lysozyme, lactoferrin, BPI and elastase (Orgentec Diagnostika GmbH; Mainz, Germany). All tests were analyzed according to the manufacturer's recommendations in a DSX System (DYNEX Technologies). The cut-off values were previously determined in 55 healthy subjects (90th percentile).

The hospital institutional review board approved the study and all patients provided written informed consent.

\section{Statistical analysis}

Descriptive statistics include $n$, percent and median with 25-75 percentiles. Differences between groups were evaluated with the Student $\mathrm{t}$ test or Mann-Whitney $\mathrm{U}$ test for continuous variables, and Chi-square test or Fisher's exact test for categorical variables. Comparisons between histologic classes were done with the KruskallWallis test. Logistic regression was used in multivariate analyses to evaluate associations between significant variables $(p \leq 0.10)$ identified 
Citation: Hinojosa-Azaola A, Nuñez-Alvarez CA, Uriarte-Hernández CM, García-Hernández JL, Alcocer-Varela J, et al. (2016) Anti-Neutrophil Cytoplasmic Antibody-Associated Vasculitis in Mexican Population: Clinical Phenotype, Autoantibody Profile and Renal Characteristics. J Vasc 2: 110. doi:10.4172/2471-9544.100110

Page 3 of 7

from bivariate analyses. OR and 95\% confidence intervals (CI) were calculated. Patient survival was assessed by life-table analysis using the Kaplan-Meier method. Differences in survival were calculated using the log-rank test. Correlations of antibodies and clinical manifestations were assessed using Spearman's correlation test with Bonferroni correction. Exact p-values are reported and a two-sided p-value of $\leq 0.05$ was considered statistically significant. All analyses were done using Stata software (Stata Corp; College Station, Texas USA), version 12.0

\section{Results}

\section{Clinical and renal histologic characteristics}

We studied 40 patients with AAV; 23 (58\%) female gender (1.3:1 female to male ratio), 35 (88\%) had diagnosis of GPA and 5 (12\%) of MPA. In 35/40 renal biopsy was performed at diagnosis of vasculitis, therefore, disease duration was 0 months (minimum 0 and maximum 43 months). Age at diagnosis was 53 years (37-58), and the most prevalent comorbidity was arterial hypertension, present in $16(41 \%)$.

Most of the patients were ANCA positive at diagnosis (98\%); CANCA staining pattern was positive in $88 \%$, while P-ANCA was observed in $18 \%$ and atypical X-ANCA in $35 \%$. PR3-ANCA positivity was present in $77 \%$ and MPO-ANCA in $17 \%$.

Disease activity according to BVAS/GPA at diagnosis was 24 (16-25), while FFS was 2 points (1-2). The most frequent clinical manifestations were general (98\%), renal (80\%), pulmonary (63\%), ear, nose and throat (ENT) (58\%). Patients had a median of 4 (2-6) organs affected according to BVAS/GPA categories.

The most prevalent specific manifestations according to each category of the BVAS/GPA were: fever $(80 \%)$, rise in creatinine/fall in creatinine clearance (78\%), hematuria (75\%), arthritis (53\%), alveolar hemorrhage $(48 \%)$, pulmonary infiltrates $(43 \%)$, nasal crusts $(38 \%)$, purpura (30\%), and sensory peripheral neuropathy (25\%).

Baseline serum creatinine was $3.9 \mathrm{mg} / \mathrm{dl}$ (2.6-4.3), eGFR $16 \mathrm{ml} / \mathrm{min}$ (11-22), and $24 \mathrm{hr}$ proteinuria $2.5 \mathrm{~g}$ (1.9-3). Five patients (13\%) were on dialysis at diagnosis, (1 with mixed and 4 with sclerotic histological class).

The most prevalent renal histological class was sclerotic (23 patients, $58 \%$ ), followed by mixed ( 13 patients, $32 \%$ ), focal ( 3 patients, $8 \%$ ) and crescentic (1 patient, 2\%). No significant differences were found between histological classes when comorbidities, clinical (including disease activity), renal, and laboratory variables were analyzed.

In all renal biopsies, independent of the histological class, percentage of normal glomeruli was $22 \%$ (11-60), tubular atrophy $30 \%$ (15-59), interstitial fibrosis 30\% (20-50) and interstitial infiltrate $15 \%$ (10-30\%).

Six patients (15\%) died during follow-up (4 with sclerotic class, 1 with focal, and 1 with mixed class). Median length of follow-up time was 24 months (minimum 6, maximum 96). Survival in patients with sclerotic class was $100 \%$ and $66 \%$ at 1 and 5 years respectively; whereas in patients with non-sclerotic class it was $93 \%$ at 1 and 5 years. No difference in overall survival was found between groups ( $\mathrm{p}=0.55 \mathrm{log}$ rank). Causes of death were infectious, pneumonia in four patients and sepsis in two. Table 1 summarizes clinical and renal characteristics at diagnosis.

\begin{tabular}{|c|c|}
\hline Characteristic & $\mathbf{N}(\%)$ \\
\hline \multicolumn{2}{|l|}{ Demographics and comorbidities } \\
\hline Female gender & $23(58)$ \\
\hline Age at diagnosis-years & $53(37-58)$ \\
\hline GPA & $35(88)$ \\
\hline MPA & $5(12)$ \\
\hline Diabetes & $3(8)$ \\
\hline Dyslipidemia & $6(15)$ \\
\hline Arterial hypertension & $16(41)$ \\
\hline Smoking & $7(18)$ \\
\hline \multicolumn{2}{|l|}{ Clinical variables } \\
\hline BVAS/GPA & $24(16-25)$ \\
\hline FFS at diagnosis & $2(1-2)$ \\
\hline General & $39(98)$ \\
\hline Cutaneous & $15(38)$ \\
\hline Mucous membranes/Eyes & $7(18)$ \\
\hline Ear, nose and throat & $23(58)$ \\
\hline Cardiovascular & 0 \\
\hline Gastrointestinal & 0 \\
\hline Pulmonary & $25(63)$ \\
\hline Renal & $32(80)$ \\
\hline Nervous system & $12(30)$ \\
\hline \multicolumn{2}{|l|}{ Renal variables } \\
\hline \multicolumn{2}{|l|}{ Renal histological class } \\
\hline Focal & $3(8)$ \\
\hline Crescentic & $1(2)$ \\
\hline Mixed & $13(32)$ \\
\hline Sclerotic & $23(58)$ \\
\hline Baseline serum creatinine-mg/dl & $3.9(2.6-4.3)$ \\
\hline eGFR (MDRD) $\mathrm{ml} / \mathrm{min}$ at diagnosis & $16(11-22)$ \\
\hline Proteinuria (gr/24 h) & $2.5(1.9-3)$ \\
\hline Protein/creatinine ratio & $2.7(2-3.2)$ \\
\hline Dialysis at diagnosis & $5(13)$ \\
\hline \multicolumn{2}{|l|}{ Laboratory variables at diagnosis } \\
\hline ANCA positive (any pattern) & $39(98)$ \\
\hline C-ANCA positive & $35(88)$ \\
\hline P-ANCA positive & $7(18)$ \\
\hline X-ANCA positive & $14(35)$ \\
\hline
\end{tabular}


Citation: Hinojosa-Azaola A, Nuñez-Alvarez CA, Uriarte-Hernández CM, García-Hernández JL, Alcocer-Varela J, et al. (2016) Anti-Neutrophil Cytoplasmic Antibody-Associated Vasculitis in Mexican Population: Clinical Phenotype, Autoantibody Profile and Renal Characteristics. J Vasc 2: 110. doi:10.4172/2471-9544.100110

Page 4 of 7

\begin{tabular}{|l|l|}
\hline Anti-PR3 positive- $\mathrm{n}+\mathrm{n}$ tested $(\%)$ & $27 / 35(77)$ \\
\hline Anti-MPO positive- $\mathrm{n}+\mathrm{n}$ tested $(\%)$ & $6 / 35(17)$ \\
\hline hsCRP-mg/l & $3(2-4.5)$ \\
\hline ESR-mm/h & $65(46-94)$ \\
\hline
\end{tabular}

Table 1: Clinical and renal characteristics at diagnosis. GPA: Granulomatosis with Polyangiitis; MPA: Microscopic Polyangiitis; BVAS/GPA: Brirmingham Vasculitis Activity Score for GPA; FFS: Five Factor Score; eGFR: Estimated Glomerular Filtration Rate; Anti-MPO: Anti-Myeloperoxidase Antibody; Anti-PR3: Anti-Proteinase 3 Antibody; hsCRP: high sensitivity C - reactive protein; ESR: Erythrocyte Sedimentation Rate.

\section{Autoantibody profile}

Fifty patients with AAV were studied transversally (40 with GPA, 4 with MPA, 3 with EGPA, and 3 with renal-limited vasculitis); 31 (62\%) were female and 19 (38\%) were male (1.6:1 ratio); age was 52 years (41-64), and disease duration was 3 years (2-5).

Disease activity according to BVAS/GPA at the time of the evaluation was 3 (minimum 0, maximum 14); ENT, neurologic and renal were the most prevalent clinical manifestations, present in $28 \%$, $24 \%$ and $16 \%$, respectively. General symptoms were present in $14 \%$, while cutaneous, mucous membranes/eyes and pulmonary manifestations were present in $\leq 10 \%$.

In patients with $\mathrm{AAV}$, serum creatinine was $1.1 \mathrm{mg} / \mathrm{dl}(0.8-1.8)$; leukocytes $7.7 \times 10^{3} / \mathrm{mm}^{3}$ (5.5-10.7); hemoglobin $13.5 \mathrm{~g} / \mathrm{dl}$ (12.3-14.6), and platelet count $262 \mathrm{~K} / \mathrm{ul}$ (212-321). Anemia was present in 9 (18\%), while leukopenia and thrombocytosis were infrequent (2\%).

At the moment of the evaluation, $41(82 \%)$ patients were receiving prednisone, dose of $10 \mathrm{mg} / \mathrm{d}$ (5-15); 28 (56\%) azathioprine, dose of 100 $\mathrm{mg} / \mathrm{d}$ (75-150); 4 (8\%) were receiving IV monthly cyclophosphamide at a dose of $875 \mathrm{mg}$ (750-1000), and 3 (6\%) methotrexate, dose of 12.5 $\mathrm{mg} /$ week (10-17.5). Median ESR and hsCRP was $7 \mathrm{~mm} / \mathrm{hr}$ (4-22), and $0.3 \mathrm{mg} / \mathrm{dl}(0.2-1.8)$, respectively.

Patients with AAV were older than controls (51.5 vs 31 years, $\mathrm{p}<0.001)$. ANCA positivity was more frequent in patients with AAV than controls $(\mathrm{p}<0.001)$. As expected by the GPA predominance over the other forms of AAV, the main ANCA IIF staining pattern was CANCA, present in $34(68 \%)$ of the patients vs $6(14 \%)$ of the controls (p $<0.001)$, followed by X-ANCA in $11(22 \%)$ of the patients and $2(5 \%)$ of controls $(\mathrm{p}<0.01)$, and P-ANCA in $9(18 \%)$ and $1(2 \%)$, respectively $(\mathrm{p}=0.01)$. Positivity for two different ANCA IIF staining patterns was observed in $11(22 \%)$ of patients and $1(2 \%)$ of controls $(\mathrm{p}=0.005)$. Seven patients (14\%) presented high ANCA titers $(>1: 160)$, compared to $5(12 \%)$ of the controls $(\mathrm{p}=0.76)$; none of the healthy subjects was ANCA positive. Twenty-tree patients (46\%) were positive for PR3ANCA, compared to $4(9 \%)$ of controls $(\mathrm{p}<0.0001)$, while highsensitivity PR3-ANCA was positive in $43(86 \%)$ of patients and in 21 $(55 \%)$ of controls $(\mathrm{p}=0.002)$, and MPO-ANCA in $9(18 \%)$ and $5(12 \%)$, respectively $(\mathrm{p}=0.56)$. In multivariate analysis, age (OR $1.08,95 \% \mathrm{CI}$ 1.03-1.13, $\mathrm{p}=0.02$ ), and ANCA positivity (OR 15, 95\% CI 3.90-57.59, $\mathrm{p}$ $<0.0001$ ) were independent variables associated with AAV.

Antibodies directed to minor targets were infrequent in our patients with AAV. Elastase, BPI, and cathepsin G were positive in 1 patient with EGPA who was C-ANCA positive; while lactoferrin was positive

in 1 GPA patient with C-ANCA and X-ANCA staining patterns, and lysozyme in none. Both patients were negative for MPO and PR3ANCA. In the control group, 1 patient with SLE was positive for elastase and cathepsin G, and 1 only for cathepsin G; 1 with infection (diabetic foot) was positive for elastase and BPI, and 1 with HIV was positive for BPI and cathepsin G. Two more patients with infections (1 with pyelonephritis and 1 with endocarditis) were positive for BPI, and 2 for elastase (1 with HIV and histoplasmosis and 1 with disseminated tuberculosis).

No significant correlations were identified between specific autoantibodies and clinical or laboratory variables. A positive correlation was found between PGA and BVAS/GPA ( $\mathrm{r}=0.83, \mathrm{p}<$ $0.0001)$, hsCRP and BVAS/GPA ( $\mathrm{r}=0.51, \mathrm{p}=0.03)$, and hsCRP and PGA $(\mathrm{r}=0.66, \mathrm{p}>0.0001)$.

Data summarizing the antibody profile in patients with AAV and controls is shown in (Table 2).

\begin{tabular}{|l|l|l|l|}
\hline Variable & Patients & Controls & $\mathbf{p}$ \\
\hline & $\mathbf{n = 5 0}$ & $\mathbf{n}=\mathbf{4 3}$ & \\
\hline Age-years & $\begin{array}{l}51.5 \\
(21-85)\end{array}$ & $\begin{array}{l}31 \\
(18-77)\end{array}$ & $<0.001$ \\
\hline Female-n (\%) & $31(62)$ & $27(63)$ & 1 \\
\hline ANCA 1:20-n (\%) & $43(86)$ & $8(19)$ & $<0.001$ \\
\hline ANCA 1:40-n (\%) & $34(68)$ & $6(14)$ & $<0.001$ \\
\hline ANCA 1:80-n (\%) & $15(30)$ & $6(14)$ & 0.08 \\
\hline ANCA > 1:160-n (\%) & $7(14)$ & $5(12)$ & 0.76 \\
\hline C-ANCA-n (\%) & $34(68)$ & $6(14)$ & $<0.001$ \\
\hline P-ANCA-n (\%) & $9(18)$ & $1(2)$ & 0.01 \\
\hline X-ANCA-n (\%) & $11(22)$ & $2(5)$ & 0.01 \\
\hline Anti-PR3 positive-n (\%) & $23(46)$ & $4(9)$ & 1 \\
\hline $\begin{array}{l}\text { Anti-PR3 high sensitive positive-n + } \\
\text { tested (\%) }\end{array}$ & $43(86)$ & $\begin{array}{l}21 / 38 \\
(55)\end{array}$ & 0.002 \\
\hline Anti-MPO positive-n (\%) & $9(18)$ & $5(12)$ & 0.56 \\
\hline Elastase positive-n +/n tested (\%) & $1(2)$ & $4 / 38(11)$ & 0.16 \\
\hline BPI positive-n+/n tested (\%) & $1(2)$ & $4 / 38(11)$ & 0.16 \\
\hline Cathepsin G positive-n +/n tested (\%) & $1(2)$ & $3 / 38(8)$ & 0.31 \\
\hline Lactoferrin positive-n (\%) & $1(2)$ & 0 & 1 \\
\hline Lysozyme positive-n (\%) & 0 & 0 & - \\
\hline
\end{tabular}

Table 2: Autoantibody profile in patients with AAV and controls. ANCA: Anti-Neutrophil Cytoplasmic Antibodies; C-ANCA: Cytoplasmic Staining Pattern; P-ANCA: Perinuclear Staining Pattern; X-ANCA: Atypical Staining Pattern; anti-PR3: anti-proteinase 3 antibody; anti-MPO: anti-myeloperoxidase antibody; BPI: Bactericidal Permeability Increasing protein. 
Citation: Hinojosa-Azaola A, Nuñez-Alvarez CA, Uriarte-Hernández CM, García-Hernández JL, Alcocer-Varela J, et al. (2016) Anti-Neutrophil Cytoplasmic Antibody-Associated Vasculitis in Mexican Population: Clinical Phenotype, Autoantibody Profile and Renal Characteristics. J Vasc 2: 110. doi:10.4172/2471-9544.100110

Page 5 of 7

\section{Discussion}

We describe the characteristics of Mexican patients with AAV in two different scenarios. In the first one, patients were analyzed at diagnosis of vasculitis, when multiple organ involvement, high disease activity and renal damage were present. At this point, the main renal histological class was sclerotic in more than half of our patients, with no differences between histological classes regarding clinical, serological manifestations and survival. Secondly, patients were assessed during disease course to evaluate the prevalence of ANCA and antibodies specific for other neutrophils antigens. C-ANCA and PR3-ANCA were the predominant profile, while the antibodies to minor targets were infrequent, without correlation with specific clinical manifestations.

Our data regarding predominance of female gender, age, and main clinical manifestations at diagnosis (ENT, lung, renal and peripheral nervous system) corresponds well with the characteristics described in a report from Latin-American (Chilean) patients with GPA and MPA, where ENT involvement was present in $38-57 \%$, lung in $28-62 \%$, renal in $68-78 \%$, and peripheral nervous system in $17-59 \%$ [18]. High frequency of constitutional symptoms $(>90 \%)$, and high serum creatinine at diagnosis $(4 \mathrm{mg} / \mathrm{dl})$ were also identified in 80 German patients with AAV [19]. It is worth pointing out the high frequency of alveolar hemorrhage at diagnosis in $48 \%$ of our patients, as defined by BVAS/GPA. Previous studies have also described high incidences of this severe manifestation [20]. Our findings of frequent ENT involvement at diagnosis and predominant chronic renal histological lesions differ from the ones reported by Rahmattulla et al. [21], where the presence of ENT involvement was associated with prognostically favorable renal biopsy findings (focal class, less interstitial fibrosis and tubular atrophy), and better renal function.

A FFS of 2 points at diagnosis was present in our patients, mostly due to the renal items, since gastrointestinal and cardiac manifestations were not observed. Despite the 5-year survival of $40 \%$ expected for this score, $66 \%$ and $93 \%$ of our patients with sclerotic and the other histological classes respectively, were alive at 5 years, similar to the 5year survival reported in patients from the Limburg Renal Registry cohort [22]. As in our study, other authors have described no significant differences in patient survival among histological classes [23]. This should be interpreted cautiously, since the purpose of our study was to assess the differences in the renal histological classes rather than to make conclusions regarding the survival and outcome of our population.

Sreih et al. reported higher frequency of renal involvement in Hispanic patients (Mexican ancestry) compared to Caucasians (85\% vs $48 \%$ ), with similar characteristics than our population regarding renal function and BVAS/GPA at presentation, but more patients with dialysis requirements ( $31.8 \%$ versus $13 \%$ in our study) [13]. The most frequent renal histological class in our analysis was sclerotic, opposite to other single-center studies and multicentric clinical trials that have validated the histological classification, where sclerotic class was absent or very infrequent $[7,22,24,25]$. A study that analyzed the histopathological classification in 156 Australian patients reported 20 cases $(17 \%)$ with sclerotic class, and concluded that the sclerotic patterns are the lesions more reliably identified by pathologists [26]. A possible explanation for the predominance of sclerotic class in our population is a delay in diagnosis or referral to specialized medical centers due to socioeconomic reasons.
The distribution of the four renal histological categories is similar in Europe, Canada, India and China, with crescentic class being the dominant, whereas in Japan the focal class predominates, and in USA the mixed $[7,23,27-33]$. The presence of impaired renal function, and the low percentage of normal glomeruli $(<25 \%)$ in our population are factors known to be associated with poor renal outcome $[25,34,35]$.

Predominance for GPA, C-ANCA and PR3-ANCA in our population is similar to the phenotype of European patients with AAV. Antibodies to minor targets were infrequent in our patients with AAV, similar to the findings reported by Talor et al. [5]; therefore, we were unable to find a correlation with specific clinical manifestations. The utility of testing for antibodies to minor antigens is to explain immunofluorescence reactivity for ANCA in the absence of MPO and PR3-ANCA positivity.

Recent focus has been directed on the study of potential biomarkers in AAV. Among the candidate's biomarkers are those related to B and T cells, markers of vascular damage or activation, and organ-specific markers. Promising biomarkers identified are MMP-2, MMP-3, MMP-9, TIMP-1, VEGF, CXCL13 (BCA-1), complement pathway, and high mobility group box 1 (HMGB1) [36,37].

The IL-33/ST2 (suppression of tumorigenicity 2) pathway has been subject of recent studies, especially in patients with cardiovascular and pulmonary diseases, but also in systemic infection and inflammation. IL-33 binds to ST2, a member of the IL-1 receptor family that mediates important Th2 functions. A recent study of 139 AAV patients and 62 controls, found that newly diagnosed AAV had higher soluble ST2 levels than controls, and these levels were significantly higher in active newly diagnosed AAV patients than in patients in remission, whereas IL-33 levels were also higher in AAV than controls, but no difference was found in active patients compared to patients in remission, suggesting a potential role for serum soluble ST2 as a biomarker of activity in AAV [38]. To date, no single biomarker has met high sensitivity or specificity to justify its clinical usefulness.

The present study has some limitations. First, the reduced number of patients, although the sample represents a single center, numbers are small. Second, our patients belong to a tertiary care center, where cases with a more severe spectrum of the disease are referred; therefore the results might not be representative of the entire Mexican population with AAV. Last, no conclusions about the predictive value of the histological classification for renal outcome can be derived from our study, since treatment, remission, and relapses were not assessed in follow-up, and due to limited number of patients in each histological class. Nevertheless, we consider that our study adds valuable information about the expression of AAV in Mexican patients, both at diagnosis and during the course of the disease with regard to clinical, serologic and renal histologic characteristics.

In conclusion, GPA was the most frequent AAV in our population; constitutional, ENT, lung and renal involvement were the predominant clinical manifestations. Sclerotic renal histological class was the most frequent, without differences between histological classes regarding clinical, serological manifestations and survival. These data suggest that our patients present severe disease activity, and high prevalence of renal involvement and damage. Prospective studies are needed to assess long-term outcomes in Mexican patients with AAV. 
Citation: Hinojosa-Azaola A, Nuñez-Alvarez CA, Uriarte-Hernández CM, García-Hernández JL, Alcocer-Varela J, et al. (2016) Anti-Neutrophil Cytoplasmic Antibody-Associated Vasculitis in Mexican Population: Clinical Phenotype, Autoantibody Profile and Renal Characteristics. J Vasc 2: 110. doi:10.4172/2471-9544.100110

Page 6 of 7

\section{Funding}

This work was supported with research funds provided by the Instituto Nacional de Ciencias Médicas y Nutrición Salvador Zubirán (INCMNSZ-AI-010).

\section{Conflicts of interest}

The authors declare no conflicts of interest.

\section{References}

1. Kobayashi S, Fujimoto S (2013) Epidemiology of vasculitides: differences between Japan, Europe and North America. Clin Exp Nephrol 17: 611-614.

2. Katsuyama T, Sada KE, Makino H (2014) Current concept and epidemiology of systemic vasculitides. Allergol Int 63: 505-513.

3. Geetha D, Poulton CJ, Hu Y, Seo P, McGregor JA, et al. (2014) Clinical characteristics and outcome of pauci-immune glomerulonephritis in African Americans. Semin Arthritis Rheum 43: 778-783.

4. Chen M, Yu F, Zhang Y, Zou WZ, Zhao MH, et al. (2005) Characteristics of Chinese patients with Wegener's granulomatosis with antimyeloperoxidase autoantibodies. Kidney Int 68: 2225-2229.

5. Talor MV, Stone JH, Stebbing J, Barin J, Rose NR, et al. (2007) Antibodies to selected minor target antigens in patients with anti-neutrophil cytoplasmic antibodies (ANCA). Clin Exp Immunol 150: 42-48.

6. Korkmaz B, Horwitz MS, Jenne DE, Gauthier F (2010) Neutrophil elastase, proteinase 3, and cathepsin $\mathrm{G}$ as therapeutic targets in human diseases. Pharmacol Rev 62: 726-759.

7. Berden AE, Ferrario F, Hagen EC, Jayne DR, Jennette JC, et al. (2010) Histopathologic classification of ANCA-associated glomerulonephritis. J Am Soc Nephrol 21: 1628-1636.

8. Rahmattulla C, Brujin JA, Bajema IM (2014) Histopahological classification of antineutrophil cytoplasmic antobody-associated glomerulonephritis: an update. Curr Opin Nephrol Hypertens 23: 224-231.

9. Córdova-Sánchez BM, Mejía-Vilet JM, Morales-Buenrostro LE, LoyolaRodriguez G, Uribe-Uribe NO, et al. (2016) Clinical presentation and outcome prediction of clinical, serological, and histopathological classification schemes in ANCA-associated vasculitis with renal involvment. Clin Rheumatol.

10. Flores-Suárez LF, Alba MA (2015) Clinical characteristics of antineutrophil cytoplasmic antibody-associated vasculitis in a respiratory diseases referral center in Mexico (1982-2010). Gac Med Mex 151:176-185.

11. Flores-Suárez LF, Villa AR (2007) Spectrum of Wegener granulomatosis in a Mexican population. Ann N Y Acad Sci 1107: 400-409.

12. Solar-Cafaggi D, Atisha-Fregoso Y, Hinojosa-Azaola A (2015) Plasmapheresis therapy in ANCA-associated vasculitides: A single-center retrospective analysis of renal outcome and mortality. J Clin Apher. doi: 10.1002/jca.21415.

13. Sreih AG, Mandhadi R, Aldaghlawi F, Khan A, Irshad V, et al. (2015) ANCA-associated vasculitis in Hispanic Americans: an unrecognized severity. Clin Rheumatol 34: 943-948.

14. Jennette JC, Falk RJ, Bacon PA, Basu N, Cid MC, et al. (2013) 2012 Revised International Chapel Hill Consensus Conference Nomenclature of Vasculitides. Arthritis Rheum 65: 1-11.

15. Stone JH, Hoffman GS, Merkel PA, Min YI, Uhlfelder ML, et al. (2001) A disease specific activity index for Wegener's granulomatosis: Modification of the Birmingham Vasculitis Activity Score. International Network for the Study of the Systemic Vasculitides (INSSYS). Arthritis Rheum 44: 912-920.

16. Guillevin L, Pagnoux C, Seror R, Mahr A, Mouthon L, et al. (2011) The Five-Factor Score revisited: assessment of prognoses of systemic necrotizing vasculitides based on the French Vasculitis Study Group (FVSG) Cohort. Medicine 90: 19-27.

17. Levey AS, Bosch JP, Lewis JB, Greene T, Rogers N, et al. (1999) A more accurate method to estimate glomerular filtration rate form serum creatinine: a new prediction equation. Ann Intern Med 130: 461-470.

18. Cisternas M, Soto L, Jacobelli S, Marinovic MA, Vargas A, et al. (2005) Clinical features of Wegener granulomatosis and microscopic polyangiitis in Chilean patients. Rev Med Chil 133: 273-278.

19. Weidner S, Geuss S, Hafezi-Rachti S, Wonka A, Rupprecht HD (2004) ANCA-associated vasculitis with renal involvement: an outcome analysis. Nephrol Dial Transplant 19: 1403-1411.

20. Haworth SJ, Savage CO, Carr D, Hughes JM, Rees AJ (1985) Pulmonary haemorrhage complicating Wegener's granulomatosis and microscopic polyarteritis. Br Med J 290:1775-1778.

21. Rahmattulla C, de Lind van Wijngaarden RA, Berden AE, Hauer HA, et al. (2015) Renal function and ear, throat involvement in anti-neutrophil cytoplasmic antibody-associated vasculitis: prospective data from the European Vasculitis Society clinical trials. Rheumatology (Oxford) 54: 899-907.

22. Hilhorst M, Wilde B, van Paassen P, Winkens B, van Breda Vriesman P, et al. (2013) Improved outcome in anti-neutrophil cytoplasmic antibody (ANCA)-associated glomerulonephritis: a 30-year follow-up study. Nephrol Dial Transplant 28: 373-379.

23. Chang DY, Wu LH, Liu G, Chen M, Kallenberg CG, et al. (2012) Reevaluation of the histopathologic classification of ANCA-associated glomerulonephritis: A study of 121 patients in a single center. Nephrol Dial Transplant 27: 2343-2349.

24. Berden AE, Jones RB, Erasmus DD, Walsh M, Noël LH, et al. (2012) Tubular lesions predict renal outcome in antineutrophil cytoplasmic antibody-associated glomerulonephritis after rituximab therapy. J Am Soc Nephrol 23: 313-321.

25. Tanna A, Guarino L, Tam FW, Rodriquez-Cubillo B, Levy JB, et al. (2015) Long-term outcome of anti-neutrophil cytoplasm antibody-associated glomerulonephritis: evaluation of the international histological classification and other prognostic factors. Nephrol Dial Transplant 30: 1185-1192.

26. Ford SL, Polkinghorne KR, Longano A, Dowling J, Dayan S, et al. (2014) Histopathologic and clinical predictors of kidney outcomes in ANCAassociated vasculitis. Am J Kidney Dis 63: 227-235.

27. Muso E, Endo T, Itabashi M, Kakita H, Iwasaki Y, et al. (2013) Evaluation of the newly proposed simplified histological classification in Japanese cohorts of myeloperoxidase-anti-neutrophil cytoplasmic antibodyassociated glomerulonephritis in comparison with other Asian and European cohorts. Clin Exp Nephrol 17: 659-662.

28. Ellis CL, Manno RL, Havill JP, Racusen LC, Geetha D (2013) Validation of the new classification of pauci-immune glomerulonephritis in a United States cohort and its correlation with renal outcome. BMC Nephrol 14: 210 .

29. Iwakiri T, Fujimoto S, Kitagawa K, Furuichi K, Yamahana J, et al. (2013) Validation of a newly porposed histopathological classification in Japanese patients with antineutrophil cytoplasmic antibody-associated glomerulonephritis. BMC Nephrol 14: 125.

30. Haas M, Rastaldi MP, Fervenza FC (2014) Histologic classification of glomerular diseases: clinicopathologic correlations, limitations exposed by validation studies, and suggestions for modification. Kidney Int 85 : 779-793.

31. Nohr E, Girard L, James M, Benediktsson H (2014) Validation of a histopathologic classification scheme for antineutrophil cytoplasmic antibody-associated glomerulonephritis. Hum Pathol 45: 1423-1429.

32. Naidu GS, Sharma A, Nada R, Kohli HS, Jha V, et al. (2014) Histopathological classification of pauci-immune glomerulonephritis and its impact on outcome. Rheumatol Int 34: 1721-1727.

33. Andreiana I, Stancu S, Avram A, Taran L, Mircescu G (2015) ANCA positive crescentic glomerulonephritis outcome in a Central East European cohort: a retrospective study. BMC Nephrol 16: 90. 
Citation: Hinojosa-Azaola A, Nuñez-Alvarez CA, Uriarte-Hernández CM, García-Hernández JL, Alcocer-Varela J, et al. (2016) Anti-Neutrophil Cytoplasmic Antibody-Associated Vasculitis in Mexican Population: Clinical Phenotype, Autoantibody Profile and Renal Characteristics. J Vasc 2: 110. doi:10.4172/2471-9544.100110

Page 7 of 7

34. Hilhorst M, Wilde B, van Breda Vriesman P, van Paassen P, Cohen Tervaert JW; Limburg Renal Registry (2013) Estimating renal survival using the ANCA-associated GN classification. J Am Soc Nephrol 24: 1371-1375.

35. Li ZY, Gou SJ, Chen M, Zhao MH (2013) Predictors for outcomes in patients with severe ANCA-associated glomerulonephritis who were dialysis-dependeny at presentation: a study of 89 cases in a single Chinese center. Semin Arthritis Rheum 42: 515-521.
36. Monach PA (2014) Biomarkers in vasculitis. Curr Opin Rheumatol 26: 24-30.

37. Perez-Alamino R, Maldonado-Ficco H (2015) New insights on biomarkers in systemic vasculitis. Curr Rheumatol Rep 17: 12.

38. Hladinova Z, Hruskova Z, Svobodova B, Malickova K, Lanska V, et al. (2015) Increased levels of soluble ST2 in patients with active newly diagnosed ANCA-associated vasculitis. Mediators Inflamm 2015: 603750. 